\title{
Recurrent dynamical symmetry breakings and restorations by Wilson lines at finite densities on a torus
}

\author{
Chung-Chieh Lee and Choon-Lin Ho \\ Department of Physics, Tamkang University, Tamsui 25137, Taiwan
}

(Jan 27, 2000)

\begin{abstract}
In this paper we derive the general expression of a one-loop effective potential of the nonintegrable phases of Wilson lines for an $\mathrm{SU}(\mathrm{N})$ gauge theory with a massless adjoint fermion defined on the spactime manifold $R^{1, d-3} \times T^{2}$ at finite temperature and fermion density. The Phase structure of the vacuum is presented for the case with $d=4$ and $N=2$ at zero temperature. It is found that gauge symmetry is broken and restored alternately as the fermion density increases, a feature not found in the Higgs mechanism. It is the manifestation of the quantum effects of the nonintegrable phases. PACS number(s): 11.10.Wx, 11.10.Kk, 11.15.Ex
\end{abstract}




\section{Introduction}

By now no one would deny that one of our most important quests is the search for the theory that unifies all the known fundamental interactions. This dream found its partial realization in the Weinberg-Salam-Glashow model of electroweak theory. From this model one learns of the ironic yet important interplay between the requirement in one's unified theory of certain gauge symmetries, and the necessity of breaking them.

One of the most original and beautiful ideas in the search of a unified theory is the assumption of the existence of extra compactified dimensions, originated from the works of Nordström, Kaluza, and Klein [1]. These compactified dimensions manifest themselves as gauge fields in flat four-dimensional spactimes. The beauty and simplicity of this idea fascinated even the great Einstein, who returned to it several times in the latter part of his life [2]. The idea, however, soon met with its demise owing to the lack of experimental evidence. It was not until the 1970s that interest in Kaluza-Klein theories were revived by modern attempts of unified theory such as the theories of supergravity and superstrings. In these modern theories, however, gauge fields are usually assumed to be present in higher dimensions. Nevertheless, it is fair to say that the principle of gauge invariance, and the assumption of extra compactified dimensions constitute the two most important ingredients in most of the modern versions of a unified theory. More recently, in a different development, the possibility of extra dimensions has also attracted the attention of particle phenomenologists [3]: it was argued that various unification scales might be lower than what were previously thought of, if extra dimensions exist.

Inspired by the Kaluza-Klein scenario, properties of gauge theories in nontrivial spacetimes have also received considerable interest during the last three decades. Of the various topics considered so far an interesting one is the study of the effects of nonintegrable phases of the Wilson lines. In a gauge theory on a multiply connected space, these nonintegrable phases become dynamical degrees of freedom «, 5, and have many interesting implications. They lead to a $\theta$-vacuum in QED on a circle [6]. On toroidal spaces they induce restriction on 
the Chern-Simons term [7], and consequently lead to multicomponent anyon wave functions and the related braid group structures [8], and quantum group symmetry [9]. Last but not least, they cause dynamical gauge symmetry breaking in non-Abelian gauge theory «, 5 .

Dynamical gauge symmetry breaking by Wilson lines in a multiply connected spacetime has been studied with considerable interests since its introduction in the 1980s, and has been employed extensively in superstring phenomenology [10. Unlike the Higgs mechanism, which relies on the nonvanishing vacuum expectation values of scalar fields, the Wilson line mechanism depends essentially on the nontrivial holonomy of the vacuum gauge field configuration arises from the topology of spacetime. An interesting feature of this mechanism is that fermion mass generation does not necessarily require symmetry to be broken, and vice versa.

However, it is generally very difficult to compute dynamically the quantum effects (in terms of the effective potentials) that determine the symmetry-breaking patterns, except for a few simple compact manifolds, such as the circles $R^{1, d-2} \times S^{1}$ [4, 11] and the torus $R^{1, d-3} \times T^{2}[12,13]$. So far no computation of the effective potential has been achieved on the Calabi-Yau manifolds which are relevant to superstring phenomenology. To gain a better understanding of the mechanism, it is therefore useful to explore various aspects of the mechanism in simple manifolds.

One interesting consideration is the inclusion of the effects of finite temperature and finite fermion density. Effect of finite temperatures on Wilson line symmetry breaking has been studied for circular [14, 15] and toroidal [16] compactified spaces previously. In [17] both the effects of the temperature and the fermion density are considered for the circular case $\left(R^{1, d-2} \times S^{1}\right)$. These studies indicate that in an $\mathrm{SU}(\mathrm{N})$ gauge theory with only gauge fields and fermions in the adjoint representation of the group, higher temperatures always restore the gauge symmetry (on the circle and torus, symmetry is not broken by this mechanism when the fermions are in the fundamental representation).

Our main purpose in this paper is to present the general expressions of the one-loop effective potentials for the $\mathrm{SU}(\mathrm{N})$ gauge fields and the adjoint fermions on the spacetime 
manifold $R^{1, d-3} \times T^{2}$, with temperature and density effects taken into account. We also consider the $d=4$ dimensional case in detail, and investigate the symmetry-breaking patterns as function of the fermion density and the size of the torus at zero temperatures. Even in this simple case an interesting feature already emerges, namely, the gauge symmetry can be broken and restored alternately as the density and/or the sizes of the torus change. This is something not noted in the Higgs mechanism.

The organization of the paper is as follows. First, we give a brief review of the essence of the Wilson line mechanism in Sec.2. Section 3 presents the derivation of the general expressions of the effective potentials. In Sec. 4 the $d=4$ dimensional case is studied numerically. Section 5 concludes the paper.

\section{Wilson lines mechanism}

In this paper we consider an $\mathrm{SU}(\mathrm{N})$ gauge theory with adjoint fermions on a $d$-dimensional spacetime manifold $R^{1, d-3} \times T^{2}$. Generalizing to the case in higher dimensional torus is straightforward. The Lagrangian is given by

$$
\mathcal{L}=-\frac{1}{2} \operatorname{Tr} F_{\mu \nu} F^{\mu \nu}+i \bar{\psi} \gamma^{\mu} D_{\mu} \psi
$$

where

$$
F_{\mu \nu}=\partial_{\mu} A_{\nu}-\partial_{\nu} A_{\mu}+i g\left[A_{\mu}, A_{\nu}\right], D_{\mu} \psi=\partial_{\mu} \psi+i g\left[A_{\mu}, \psi\right]
$$

As the space is multiplyconnected, one must specify the boundary conditions of the fields for the two noncontractible loops. Let $x^{\alpha}(\alpha=0,1, \ldots, d-3)$ and $y^{a}(a=1,2)$ label the Minkowski and the toroidal coordinates, respectively, $(\mu, \nu$ run over both $\alpha$ and $a)$. The boundary conditions are

$$
\begin{aligned}
& A_{\mu}\left(x^{\alpha}, y^{a}+L_{a}\right)=U_{a} A_{\mu}\left(x^{\alpha}, y^{a}\right) U_{a}^{\dagger}, \\
& \psi\left(x^{\alpha}, y^{a}+L_{a}\right)=e^{i \beta_{a}} U_{a} \psi\left(x^{\alpha}, y^{a}\right) U_{a}^{\dagger} .
\end{aligned}
$$


Here $L_{a}$ are the lengths of the circumferences of $T^{2}$. The phases $e^{i \beta_{a}}$ represent the continuous spin structure of the manifold when using Dirac fermions. These phases cancel from physical operators constructed bilinearly in $\bar{\psi}$ and $\psi$, but contribute to the boundary conditions.

One can then specify a particular $\mathrm{SU}(\mathrm{N})$ bundle over the torus, and determine the vacuum configuration of the connection by evaluating the effective potential for $\left\langle A_{\mu}\right\rangle$. However, in this paper, as in [12], we will confine ourselves to the trivial bundle with $\left\langle A_{\mu}\right\rangle$ a constant element of $\mathrm{SU}(\mathrm{N})$. Even this simple case gives interesting and nontrivial results on the vacuum structure in the theory.

Suppose that $\left\langle F_{\mu \nu}\right\rangle=0$ in the vacuum and therefore

$$
\left\langle A_{\mu}\right\rangle=-\frac{i}{g} V^{\dagger} \partial_{\mu} V
$$

This, in general, is physically distinct from $\left\langle A_{\mu}\right\rangle=0$ in a multiplyconnected space, since there is no gauge transformation which connects these two configurations without spoiling the boundary conditions. $V(x, y)$, which is undetermined in classical theory, is determined by quantum effects as a function of the boundary conditions $\left(U_{a}, \beta_{a}\right)$ up to a global gauge transformation. When the vacuum gauge configuration $\left\langle A_{\mu}\right\rangle$ is transformed to $\left\langle A_{\mu}^{\prime}\right\rangle=0$, the boundary conditions $U_{a}$ are rotated to

$$
U_{a}^{s y m}=V\left(x^{\alpha}, y^{a}+L_{a}\right) U_{a} V^{\dagger}\left(x^{\alpha}, y^{a}\right)
$$

As $U_{a}^{s y m}$ are the boundary condition matrices in the gauge $\left\langle A_{\mu}^{\prime}\right\rangle=0$, the residual gauge symmetry of the theory is generated by those generators of the group which commute with $U_{a}^{\text {sym }}$. This viewpoint of symmetry breaking was stressed by Witten [18]. One must, however, be reminded that the above argument is valid only for $d \geq 3$. In one-dimensional space $(d=2), A_{\mu}$ does not take on definite values because of quantum fluctuations, and therefore $V$ and $U_{a}^{s y m}$ are not well defined.

We note that $U_{a}^{s y m}$ are related to the path-ordered Wilson line integrals

$$
W_{a}(x, y)=P \exp i g \int_{y^{a}}^{y^{a}+L_{a}} A \cdot d x U_{a} .
$$

in that they have the same eigenvalues $e^{i \theta_{i}^{a}}$, the nonintegrable phases. These nonintegrable phases arise only in a topologically nontrivial space and cannot be gauged away; their values 
are determined dynamically. The restriction to flat connections greatly constrains the form of $\left\langle A_{a}\right\rangle$ on the torus (we assume $\left\langle A_{\alpha}\right\rangle=0$ on the Minkowski space), since all components must commute. Hence in general $\left\langle A_{a}\right\rangle$ is a diagonal constant element of $\mathrm{SU}(\mathrm{N})$, and we define

$$
\left\langle A_{a}\right\rangle_{i j}=\frac{1}{g L} \theta_{i}^{a} \delta_{i j}, \quad \sum_{i=1}^{N} \theta_{i}^{a}=0
$$

where $i, j$ are the $\mathrm{SU}(\mathrm{N})$ matrix indices.

One must evaluate the effective potential for $\left\langle A_{\mu}\right\rangle$ (or equivalently for the nonintegrable phase $\theta_{i}^{a}$ ) in order to determine the residual gauge symmetry of the system. We compute the one-loop effective potentials. For this purpose, it is convenient to take the background gauge with the gauge-fixing function

$$
\begin{aligned}
F[A] & =D_{\mu}[\langle A\rangle] A^{\mu} \\
& =\partial_{\mu} A^{\mu}+i g\left[\left\langle A_{\mu}\right\rangle, A^{\mu}\right] \\
& =0,
\end{aligned}
$$

and the gauge parameter $\alpha=1$. After performing an analytical continuation to Euclidean spacetime, one obtains the one-loop effective potential for the gauge and ghost fields

$$
V_{e f f}^{g+g h}=+\frac{d-2}{2} \ln \operatorname{det}\left(-D^{2}\right)
$$

and for the massless Dirac fermions

$$
V_{e f f}^{a d}=-\frac{f(d)}{2} \ln \operatorname{det}\left(-D^{2}\right)
$$

where $f(d) \equiv 2^{[d / 2]}$ is the number of components of a Dirac fermion. Here $[x]$ is the integral part of $x$. In the vacuum configuration (6), the operators $-D^{2}$ are given by

$$
\left[-D^{2}\right]_{j k}=-\partial_{\alpha} \partial^{\alpha}-\sum_{a=1}^{2}\left[\partial_{a}+\frac{i}{L_{a}}\left(\theta_{j}^{a}-\theta_{k}^{a}\right)\right]^{2} .
$$

We note here that the toroidal components of $-D^{2}$ act as mass terms in the Minkowski space. The effective potentials (8) and (9) can be evaluated by the zeta function method [12, 15, 17], according to which one has

$$
\ln \operatorname{det}\left(-D^{2}\right)=-\zeta^{\prime}(0)
$$


where the zeta function $\zeta(s)$ is defined according to the field contents. The next section is devoted to the evaluation of the effective potentials.

\section{The one-loop effective potentials}

The one-loop effective potentials at finite temperature and density can be evaluated according to the standard techniques in finite-temperature field theory [19]. The imaginary-time formalism appropriate for the study of thermal equilibrium properties is adopted here. In this formalism the time coordinate is Wick rotated to the Euclidean time $\tau=i t$. The real time in the time-evolution operator $\exp (-i t H)$ for a Hamiltonian $H$ is then related, by analytic continuation, to the inverse temperature $\beta=1 / T$ in the Boltzmann factor $\exp (-\beta H)$. The (anti-)commutativity of bosonic (fermionic) fields then requires the fields be (anti-)periodic in $\beta$. The prescription of deriving the effective potential of fermions at finite density is to modify the Euclidean time derivative $\partial_{\tau}$ by $\partial_{\tau} \rightarrow \partial_{\tau}-i \mu$, where $\mu$ is the chemical potential of the fermions. In the following sections, we present the results of applying the above techniques to the evaluation of Eqs.(8) and (9).

\subsection{Gauge and ghost fields}

Evaluation of the one-loop effective potential of the gauge and ghost fields is comparatively easy by following the steps in [15].

The zeta function for the gauge and ghost fields is

$$
\begin{aligned}
\zeta^{g+g h}(s) & =\frac{S_{d-3}}{\Gamma(s)} \int_{0}^{\infty} d t t^{s-1} \int_{0}^{\infty} \frac{d p p^{d-4}}{(2 \pi)^{d-3}} \frac{1}{\beta L_{1} L_{2}} \sum_{j, k} \sum_{n, m_{1}, m_{2}=-\infty}^{\infty} \\
& \times \exp \left[-t\left\{p^{2}+\left[\frac{2 \pi}{\beta} n\right]^{2}+\left[\frac{1}{L_{1}}\left(2 \pi m_{1}+\bar{\theta}_{j k}^{1}\right)\right]^{2}+\left[\frac{1}{L_{2}}\left(2 \pi m_{2}+\bar{\theta}_{j k}^{2}\right)\right]^{2}\right\}\right]
\end{aligned}
$$

Here $\bar{\theta}_{j k}^{a} \equiv \theta_{j}^{a}-\theta_{k}^{a}(a=1,2), S_{d-3} \equiv 2 \pi^{(d-3) / 2} / \Gamma((d-3) / 2)$ is the surface area of a $(d-3)$ dimensional unit sphere, $\beta=1 / T$ is the inverse temperature, and $t$ is a dummy integration parameter not to be confused with the time coordinate. 
One can perform the $p$ and $t$ integrations in Eq.(12) using the identity

$$
\sum_{m_{a}=-\infty}^{\infty} \exp \left\{-t\left[\frac{1}{L_{a}}\left(2 \pi m_{a}+\bar{\theta}_{j k}^{a}\right)\right]^{2}\right\}=\frac{L_{a} t^{-\frac{1}{2}}}{\sqrt{4 \pi}} \sum_{m_{a}=-\infty}^{\infty} \exp \left(-\frac{L_{a}^{2} m_{a}^{2}}{4 t}+i m_{a} \bar{\theta}_{j k}^{a}\right),
$$

which can be proven by means of the Poisson sum formula. Keeping only the finite part as $s \rightarrow 0$, one arrives at the effective potential for the gauge and ghost fields:

$$
\begin{aligned}
V_{e f f}^{g+g h}(T) & =-\frac{(d-2) \Gamma(d / 2)}{\pi^{d / 2}} \sum_{j, k}\left\{\sum_{m_{1}=1}^{\infty} \frac{\cos \left(m_{1} \bar{\theta}_{j k}^{1}\right)}{L_{1}^{d} m_{1}^{d}}+\sum_{m_{2}=1}^{\infty} \frac{\cos \left(m_{2} \bar{\theta}_{j k}^{2}\right)}{L_{2}^{d} m_{2}^{d}}\right. \\
& +2 \sum_{m_{1}=1}^{\infty} \sum_{m_{2}=1}^{\infty} \frac{\cos \left(m_{1} \bar{\theta}_{j k}^{1}\right) \cos \left(m_{2} \bar{\theta}_{j k}^{2}\right)}{\left(L_{1}^{2} m_{1}^{2}+L_{2}^{2} m_{2}^{2}\right)^{d / 2}} \\
& +2 \sum_{n=1}^{\infty}\left[\sum_{m_{1}=1}^{\infty} \frac{\cos \left(m_{1} \bar{\theta}_{j k}^{1}\right)}{\left(\beta^{2} n^{2}+L_{1}^{2} m_{1}^{2}\right)^{d / 2}}+\sum_{m_{2}=1}^{\infty} \frac{\cos \left(m_{2} \bar{\theta}_{j k}^{2}\right)}{\left(\beta^{2} n^{2}+L_{2}^{2} m_{2}^{2}\right)^{d / 2}}\right. \\
& \left.\left.+2 \sum_{m_{1}=1}^{\infty} \sum_{m_{2}=1}^{\infty} \frac{\cos \left(m_{1} \bar{\theta}_{j k}^{1}\right) \cos \left(m_{2} \bar{\theta}_{j k}^{2}\right)}{\left(\beta^{2} n^{2}+L_{1}^{2} m_{1}^{2}+L_{2}^{2} m_{2}^{2}\right)^{d / 2}}\right]\right\} .
\end{aligned}
$$

\subsection{Adjoint fermionic field}

The zeta function for the adjoint fermion fields is

$$
\begin{aligned}
\zeta^{a d}(s)= & \frac{S_{d-3}}{\Gamma(s)} \int_{0}^{\infty} d t t^{s-1} \int_{0}^{\infty} \frac{d p p^{d-4}}{(2 \pi)^{d-3}} \frac{1}{\beta L_{1} L_{2}} \\
& \times \sum_{j, k} \sum_{n, m_{1}, m_{2}=-\infty}^{\infty} \exp \left[-t\left\{p^{2}+\left[\frac{2 \pi}{\beta}\left(n+\frac{1}{2}\right)+i \mu\right]^{2}\right.\right. \\
+ & {\left.\left.\left[\frac{1}{L_{1}}\left(2 \pi m_{1}+\hat{\theta}_{j k}^{1}\right)\right]^{2}+\left[\frac{1}{L_{2}}\left(2 \pi m_{2}+\hat{\theta}_{j k}^{2}\right)\right]^{2}\right\}\right] }
\end{aligned}
$$

where $\mu$ is the chemical potential of the fermions, and $\hat{\theta}_{j k}^{a} \equiv \theta_{j}^{a}-\theta_{k}^{a}-\beta_{a}(a=1,2)$. Evaluation of this zeta function is done by generalizing the steps in [20, 17].

Performing the $p$ integration with the help of Eq.(13) and the identity

$$
\sum_{n=-\infty}^{\infty} \exp \left\{-t\left[\frac{2 \pi}{\beta}\left(n+\frac{1}{2}\right)+i \mu\right]^{2}\right\}=\frac{\beta}{\sqrt{4 \pi}} t^{-\frac{1}{2}}\left[1+2 \sum_{n=1}^{\infty}(-1)^{n} \cosh (n \beta \mu) e^{-\frac{\beta^{2} n^{2}}{4 t}}\right]
$$

one obtains the finite part of the effective potential as follows:

$$
\begin{aligned}
V_{e f f}^{a d}= & \frac{f(d)}{2^{d-1} \pi^{\frac{d-2}{2}} L_{1} L_{2}} \int_{0}^{\infty} d t t^{-\frac{d}{2}} \\
& \times \sum_{j, k}\left\{\sum_{m_{1}, m_{2}=-\infty}^{\infty} \exp \left[-t\left\{\left[\frac{1}{L_{1}}\left(2 \pi m_{1}+\hat{\theta}_{j k}^{(1)}\right)\right]^{2}+\left[\frac{1}{L_{2}}\left(2 \pi m_{2}+\hat{\theta}_{j k}^{(2)}\right)\right]^{2}\right\}\right]\right.
\end{aligned}
$$




$$
\begin{gathered}
+2 \sum_{n=1}^{\infty}(-1)^{n} \cosh (n \beta \mu) \sum_{m_{1}, m_{2}=-\infty}^{\infty} \exp \left[-t\left\{\left[\frac{1}{L_{1}}\left(2 \pi m_{1}+\hat{\theta}_{j k}^{(1)}\right)\right]^{2}\right.\right. \\
\left.\left.\left.+\left[\frac{1}{L_{2}}\left(2 \pi m_{2}+\hat{\theta}_{j k}^{(2)}\right)\right]^{2}\right\}-\frac{\beta^{2} n^{2}}{4 t}\right]\right\} .
\end{gathered}
$$

The first term on the right-hand side (rhs) of Eq.(17) is independent of the temperature $T$.

Making use of the identity

$$
\int_{0}^{\infty} d t t^{-\frac{d}{2}-1} e^{-t M^{2}-\frac{\beta^{2} n^{2}}{4 t}}=2\left(\frac{2 M}{n \beta}\right)^{\frac{d}{2}} K_{d / 2}(n \beta M)
$$

we now integrate Eq. (17) with respect to $t$ to get

$$
\begin{aligned}
& V_{e f f}^{a d}(T, \mu)= \\
& \frac{f(d) \Gamma(d / 2)}{\pi^{d / 2}} \sum_{j, k} \quad\left\{\sum_{m_{1}=1}^{\infty} \frac{\cos \left(m_{1} \hat{\theta}^{1}\right)}{L_{1}^{d} m_{1}^{d}}+\sum_{m_{2}=1}^{\infty} \frac{\cos \left(m_{2} \hat{\theta}^{2}\right)}{L_{2}^{d} m_{2}^{d}}+2 \sum_{m_{1}=1}^{\infty} \sum_{m_{2}=1}^{\infty} \frac{\cos \left(m_{1} \hat{\theta}^{1}\right) \cos \left(m_{2} \hat{\theta}^{2}\right)}{\left(L_{1}^{2} m_{1}^{2}+L_{2}^{2} m_{2}^{2}\right)^{d / 2}}\right\} \\
& +\frac{f(d)}{2^{d-3} \pi^{(d-2) / 2} L_{1} L_{2}} \sum_{j, k} \sum_{n=1}^{\infty}(-1)^{n} \cosh (n \beta \mu) \\
& \times \sum_{m_{1}, m_{2}=-\infty}^{\infty}\left(\frac{2 M_{j k}^{m_{1} m_{2}}}{n \beta}\right)^{(d-2) / 2} K_{(d-2) / 2}\left(n \beta M_{j k}^{m_{1} m_{2}}\right),
\end{aligned}
$$

where

$$
M_{j k}^{m_{1} m_{2}}=\left\{\left[\frac{2 \pi m_{1}+\hat{\theta}^{1}}{L_{1}}\right]^{2}+\left[\frac{2 \pi m_{2}+\hat{\theta}^{2}}{L_{2}}\right]^{2}\right\}^{\frac{1}{2}} .
$$

This is the general expression of the effective potential for the adjoint fermion fields at finite temperature and density.

To facilitate the numerical analysis of the symmetry-breaking patterns, it is realized that a different representation of the femionic effective potential is desirable. To this end we shall transform Eq. (19) into an integral form by using the following integral representation of the modified Bessel function:

$$
K_{\nu}(z)=\frac{\sqrt{\pi}}{\Gamma\left(\nu+\frac{1}{2}\right)}\left(\frac{z}{2}\right)^{\nu} \int_{1}^{\infty} e^{-z x}\left(x^{2}-1\right)^{\nu-\frac{1}{2}} d x ; \operatorname{Re}(z)>0, \operatorname{Re}(\nu)>-\frac{1}{2}
$$

and summing over $n$. This leads to

$$
V_{e f f}^{a d}(T, \mu)=\frac{f(d) \Gamma(d / 2)}{\pi^{d / 2}} \sum_{j, k}\left[\sum_{m_{1}=1}^{\infty} \frac{\cos \left(m_{1} \hat{\theta}^{1}\right)}{L_{1}^{d} m_{1}^{d}}+\sum_{m_{2}=1}^{\infty} \frac{\cos \left(m_{2} \hat{\theta}^{2}\right)}{L_{2}^{d} m_{2}^{d}}\right.
$$




$$
\begin{aligned}
& \left.+\quad 2 \sum_{m_{1}=1}^{\infty} \sum_{m_{2}=1}^{\infty} \frac{\cos \left(m_{1} \hat{\theta}^{1}\right) \cos \left(m_{2} \hat{\theta}^{2}\right)}{\left(L_{1}^{2} m_{1}^{2}+L_{2}^{2} m_{2}^{2}\right)^{d / 2}}\right]-\frac{f(d)}{2^{d-2} \pi^{(d-3) / 2} \Gamma\left(\frac{d-1}{2}\right)} \frac{1}{L_{1} L_{2}} \\
& \quad \times \sum_{j, k} \sum_{m_{1}, m_{2}=-\infty}^{\infty}\left(M_{j k}^{m_{1} m_{2}}\right)^{d-2}\left[\int_{1}^{\infty} \frac{\left(x^{2}-1\right)^{\frac{d-3}{2}}}{e^{\beta\left(x M_{j k}^{m_{1} m_{2}}-\mu\right)}+1} d x+(\mu \rightarrow-\mu)\right]
\end{aligned}
$$

Equation (22) is valid for those values of $m_{1}, m_{2}$ such that $M_{j k}^{m_{1} m_{2}} \neq 0$. In the case where $M_{j k}^{m_{1} m_{2}}=0$, a different representation is in order. In this case, the terms with these particular sets of $\left\{m_{1}, m_{2}\right\}$ in the second term of the rhs. of Wq. (17) become

$$
\begin{aligned}
& \frac{f(d)}{2^{d-2} \pi^{\frac{d-2}{2}}} \frac{1}{L_{1} L_{2}} \sum_{n=1}^{\infty}(-1)^{n} \cosh (n \beta \mu) \int_{0}^{\infty} d t t^{-\frac{d}{2}} e^{-\frac{\beta^{2} n^{2}}{4 t}} \\
= & \frac{f(d)}{2} \frac{\Gamma\left(\frac{d-2}{2}\right)}{\pi^{\frac{d-2}{2}}} \frac{1}{L_{1} L_{2} \beta^{d-2}}\left[\sum_{n=1}^{\infty} \frac{\left(-e^{\beta \mu}\right)^{n}}{n^{d-2}}+\sum_{n=1}^{\infty} \frac{\left(-e^{-\beta \mu}\right)^{n}}{n^{d-2}}\right] \\
= & \frac{f(d)}{2} \frac{\Gamma\left(\frac{d-2}{2}\right)}{\pi^{\frac{d-2}{2}}} \frac{1}{L_{1} L_{2} \beta^{d-2}}\left[\operatorname{Li}_{d-2}\left(-e^{\beta \mu}\right)+\operatorname{Li}_{d-2}\left(-e^{-\beta \mu}\right)\right],
\end{aligned}
$$

where $\operatorname{Li}_{s}(x)=\sum_{n=1}^{\infty} x^{n} / n^{s}$ is the polylogarithmic function of order $s$ [21]. This result can also be obtained by using the asymptotic form of the modified Bessel function

$$
K_{\nu}(x) \approx 2^{\nu-1} \Gamma(\nu) \frac{1}{x^{\nu}}, \quad x \rightarrow 0^{+}
$$

in Eqs.(19) for those terms with $M_{j k}^{m_{1} m_{2}}=0$ [17]. To ensure better convergence in numerical computation, we express the term $\operatorname{Li}_{d-2}\left(-e^{\beta \mu}\right)$ in terms of $\operatorname{Li}_{d-2}\left(-e^{-\beta \mu}\right)$ by means of the identities 21]

$$
\operatorname{Li}_{s}(-x)+(-1)^{s} \operatorname{Li}_{s}(-1 / x)=-\frac{1}{s !} \ln ^{s}(x)+2 \sum_{r=1}^{[s / 2]} \frac{\ln ^{s-2 r}(x)}{(s-2 r) !} \operatorname{Li}_{2 r}(-1)
$$

and

$$
\operatorname{Li}_{2 r}(-1)=-\frac{\left(2^{2 r-1}-1\right)}{(2 r) !} \pi^{2 r} B_{r}
$$

Here $B_{r}$ are the Bernoulli numbers.

\section{$4 S U(2)$ Gauge Theory on $R^{1,1} \times T^{2}$ Manifold $(T=0, \mu \neq$ 0)}

With the expressions of the effective potentials of the gauge fields, the ghost fields, and the adjoint fermions given in the last section, we can study the symmetry structures of the 
vacuum at different temperatures and densities by looking at the values of the nonintegrable phases $\theta_{j}^{a}$ which minimize the total effective potential $V_{e f f}=V_{e f f}^{g+g h}+V_{e f f}^{a d}$. As the expressions are rather complicated, the computational task is quite involved for large dimensionality $d$ and gauge group $\mathrm{SU}(\mathrm{N})$. Interesting features, however, already surface even for small $d$ and $N$. In this section, we study the symmetry patterns of an $\mathrm{SU}(2)$ gauge theory with adjoint fermions in $d=4$ dimensions numerically with finite densities at zero temperatures. The system with zero density and finite temperature were considered previously in [16]. Symmetry is always restored at high temperatures. We see that as the fermion density increases, the gauge symmetry of the system is broken and restored alternately.

In the case of $\mathrm{SU}(2)$ theory, there are only two independent nonintegrable phases owing to the traceless conditions:

$$
\theta_{1}^{a}=-\theta_{2}^{a}=\theta^{a} \quad, \quad a=1,2
$$

For convenience, we set $r \equiv L_{2} / L_{1}$.

The effective potential for the gauge and ghost fields with fixed $\left(\theta^{a}, r\right)$ now reads

$$
\begin{aligned}
V_{e f f}^{g+g h}\left(\theta^{a}, r\right)= & -\frac{4}{\pi^{2} L_{1}^{4}}\left\{\sum_{m_{1}=1}^{\infty} \frac{\cos 2 m_{1} \theta^{1}}{m_{1}^{4}}+\sum_{m_{2}=1}^{\infty} \frac{\cos 2 m_{2} \theta^{2}}{r^{4} m_{2}^{4}}\right. \\
& \left.+2 \sum_{m_{1}=1}^{\infty} \sum_{m_{2}=1}^{\infty} \frac{\cos \left(2 m_{1} \theta^{1}\right) \cos \left(2 m_{2} \theta^{2}\right)}{\left(m_{1}^{2}+r^{2} m_{2}^{2}\right)^{2}}\right\} .
\end{aligned}
$$

For the fermionic fields, we may use

$$
\frac{1}{e^{\beta x}+1} \rightarrow \theta(-x)
$$

where $\theta(x)$ is the Heaviside step function, to get

$$
\begin{aligned}
V_{e f f}^{a d} & =\frac{4}{\pi^{2} L_{1}^{4}}\left\{\left[\sum_{m_{1}=1}^{\infty}\left(\frac{\cos m_{1}\left(2 \theta^{1}-\beta_{1}\right)}{m_{1}^{4}}+\frac{\cos m_{1}\left(2 \theta^{1}+\beta_{1}\right)}{m_{1}^{4}}\right)\right.\right. \\
& +\sum_{m_{2}=1}^{\infty}\left(\frac{\cos m_{2}\left(2 \theta^{2}-\beta_{2}\right)}{r^{4} m_{2}^{4}}+\frac{\cos m_{2}\left(2 \theta^{2}+\beta_{2}\right)}{r^{4} m_{2}^{4}}\right) \\
& +2 \sum_{m_{1}=1}^{\infty} \sum_{m_{2}=1}^{\infty}\left(\frac{\cos m_{1}\left(2 \theta^{1}-\beta_{1}\right) \cos m_{2}\left(2 \theta^{2}-\beta_{2}\right)}{\left(m_{1}^{2}+r^{2} m_{2}^{2}\right)^{2}}\right. \\
& \left.\left.+\frac{\cos m_{1}\left(2 \theta^{1}+\beta_{1}\right) \cos m_{2}\left(2 \theta^{2}+\beta_{2}\right)}{\left(m_{1}^{2}+r^{2} m_{2}^{2}\right)^{2}}\right)\right]
\end{aligned}
$$




$$
\begin{aligned}
& -\frac{\pi}{2 r}\left[\sum _ { m _ { 1 } = 0 } ^ { \infty } \sum _ { m _ { 2 } = 0 } ^ { \infty } \left(\left[\left(2 \pi m_{1}+2 \theta^{1}-\beta_{1}\right)^{2}+\left(\frac{2 \pi m_{2}+2 \theta^{2}-\beta_{2}}{r}\right)^{2}\right] \int_{1}^{\frac{\mu L_{1}}{\sqrt{[\cdots]}}}\left(x^{2}-1\right)^{\frac{1}{2}} d x\right.\right. \\
& \left.+\left[\left(2 \pi m_{1}-2 \theta^{1}-\beta_{1}\right)^{2}+\left(\frac{2 \pi m_{2}-2 \theta^{2}-\beta_{2}}{r}\right)^{2}\right] \int_{1}^{\frac{\mu L_{1}}{\sqrt{[\cdots]}}}\left(x^{2}-1\right)^{\frac{1}{2}} d x\right) \\
& +\sum_{m_{1}=1}^{\infty} \sum_{m_{2}=0}^{\infty}\left(\left[\left(2 \pi m_{1}-2 \theta^{1}+\beta_{1}\right)^{2}+\left(\frac{2 \pi m_{2}+2 \theta^{2}-\beta_{2}}{r}\right)^{2}\right] \int_{1}^{\frac{\mu L_{1}}{\sqrt{[\cdots]}}}\left(x^{2}-1\right)^{\frac{1}{2}} d x\right. \\
& +\left[\left(2 \pi m_{1}+2 \theta^{1}+\beta_{1}\right)^{2}+\left(\frac{2 \pi m_{2}-2 \theta^{2}-\beta_{2}}{r}\right)^{2}\right] \int_{1}^{\frac{\mu L_{1}}{\sqrt{[\cdots]}}}\left(x^{2}-1\right)^{\frac{1}{2}} d x \\
& +\sum_{m_{1}=0}^{\infty} \sum_{m_{2}=1}^{\infty}\left(\left[\left(2 \pi m_{1}+2 \theta^{1}-\beta_{1}\right)^{2}+\left(\frac{2 \pi m_{2}-2 \theta^{2}+\beta_{2}}{r}\right)^{2}\right] \int_{1}^{\frac{\mu L_{1}}{\sqrt{[\cdots]}}}\left(x^{2}-1\right)^{\frac{1}{2}} d x\right. \\
& \left.+\left[\left(2 \pi m_{1}-2 \theta^{1}-\beta_{1}\right)^{2}+\left(\frac{2 \pi m_{2}+2 \theta^{2}+\beta_{2}}{r}\right)^{2}\right] \int_{1}^{\frac{\mu L_{1}}{\sqrt{[\cdots]}}}\left(x^{2}-1\right)^{\frac{1}{2}} d x\right) \\
& +\sum_{m_{1}=1}^{\infty} \sum_{m_{2}=1}^{\infty}\left(\left[\left(2 \pi m_{1}-2 \theta^{1}+\beta_{1}\right)^{2}+\left(\frac{2 \pi m_{2}-2 \theta^{2}+\beta_{2}}{r}\right)^{2}\right] \int_{1}^{\frac{\mu L_{1}}{\sqrt{[\cdots]}}}\left(x^{2}-1\right)^{\frac{1}{2}} d x\right. \\
& \left.+\left[\left(2 \pi m_{1}+2 \theta^{1}+\beta_{1}\right)^{2}+\left(\frac{2 \pi m_{2}+2 \theta^{2}+\beta_{2}}{r}-1\right)^{\frac{1}{2}} d x\right)\right]
\end{aligned}
$$

The symbol [...] in the upper limit of each integral in Eq. (30) represents the factor inside the square bracket immediately in front of the respective integral. In case this factor equals zero, and the corresponding term in Eq.(30) is replaced by

$$
-\frac{\mu^{2}}{\pi L_{1}^{2} r}
$$

obtained from Eq. (23).

For simplicity we only consider the boundary conditions $\beta_{1}=\beta_{2}=0$. The vacuum of the system at fixed $\mu$ and $r$ is determined by finding the values of the nonintegrable phases $\left(\theta^{1}, \theta^{2}\right)$ which correspond to the global minimum of the total one-loop effective potential $V_{\text {eff }}$. These values of the nonintegrable phases in turn, through the Wilson line integrals, determine the residual gauge symmetry of the system, as described in Sect. 2. Therefore, the vacuum and its symmetry are determined dynamically. In the present case, the global minima are found to be located at one of the following sets of the possible values of $\theta^{a}$ :

$$
\left(\theta^{1}, \theta^{2}\right)=(0,0),(0, \pi / 2),(\pi / 2,0) \text { or }(\pi / 2, \pi / 2)(\bmod \pi)
$$


Of these locations only the case $(0,0)(\bmod \pi)$ represents unbroken $\mathrm{SU}(2)$ symmetry. We present the results as a phase diagram with $\mu L_{1}$ versus $r=L_{2} / L_{1}$ in Fig.1. One sees that the gauge symmetry is broken and restored alternately as the fermion density $\mu$ increases at fixed $r$, or as the size of the torus changes at fixed density. This is a new feature not noticed so far in the Higgs mechanism, and is the manifestation of the quantum effect of the nonintegrable phases $\theta^{a}$. In the usual Higgs models, gauge symmetry is usually restored at zero temperature as the fermion density increases [22]. Only in gauge theories with neutral currents could an increase in fermion density increase the symmetry breaking [23]. However, no Higgs model, as far as we know, exhibits such a recurrent pattern of symmetry breaking and restoration. Implications of this feature of the Wilson line mechanism in particle and string phenomenology, and in astroparticle physics have yet to be explored.

In the limit $r=L_{2} / L_{1} \rightarrow \infty$, the spacetime $R^{1,1} \times T^{2}$ becomes the manifold $R^{1,2} \times S^{1}$. From Fig. 1 we see that the recurrent pattern of symmetry breakings and restorations persists in this limit. Wilson lines symmetry breaking on the spacetime $R^{1,2} \times S^{1}$ was studied in [17] at finite temperatures and densities. The recurrent pattern of symmetry breakings and restorations were, however, over looked in [17] as we did not extend the range of $\mu$ far enough. We therefore take this opportunity to present in Fig. 2 the correct phase diagram for the $\mathrm{SU}(2)$ theory with the boundary condition $\beta_{1}=0$ at finite temperatures and densities. For future reference we record here the first few critical values of $\mu$ at $T=0$ that define the boundaries of symmetric and broken phases: $\mu_{c} L_{1}(T=0)=1.979,6.146,9.140,12.495,15.55, \ldots \ldots$

\section{Conclusions}

In this paper we discuss Wilson line symmetry breaking of $\mathrm{SU}(\mathrm{N})$ gauge theory with adjoint fermions on the $d$-dimensional spacetime $R^{1, d-3} \times T^{2}$. General expressions of the one-loop effective potentials of the gauge and ghost fields, and the adjoint fermion fields were pre-

sented. Symmetry patterns of the vacuum structure in a $d=4$ dimensional SU(2) theory at zero temperature is considered in detail. It is noted in this case that the gauge symmetry 
can be broken and restored alternately as the fermion density changes. We expect this result to be true for all $\mathrm{SU}(\mathrm{N})$ groups and dimensionality $d$. This is a new feature not observed in the Higgs mechanism, and is the manifestation of the quantum effects of the nonintegrable phases.

\section{ACKNOWLEDGMENT}

This work was supported in part by the Republic of China through Grant No. NSC 89-2112-M-032-004.

\section{References}

[1] For a collection of some important papers on the Kaluza-Klein theories, see e.g.,: Modern Kaluza-Klein Theories, edited by T. Appelquist, A. Chodos, and P.T.O. Freund (Addison-Wesley, Reading, MA, 1987).

[2] A. Einstein and P.G. Bergmann, Ann. Math. 39, 683 (1938) (collected in [1]).

[3] N. Arkani-Hamed, S. Dimopoulous, and G. Dvali, Phys. Lett. B 429, 263 (1998).

[4] Y. Hosotani, Phys. Lett. 126B , 309 (1983); Ann. Phys. (N.Y.) 190, 233 (1989).

[5] D. Toms, Phys. Lett. 126B , 445 (1983).

[6] N.S. Manton, Ann. Phys. (N.Y.) 159, 220 (1985); J.E. Hetrick and Y. Hosotani, Phys. Rev. D38, 2621 (1988).

[7] Y. Hosotani, Phys. Rev. Lett. 62, 2785 (1989); S. Elitzer, G. Moore, A. Schwimmer and N. Seiberg, Nucl. Phys. B 326108 (1989); A.P. Polychronakos, Phys. Lett. B 241, 37 (1990). 
[8] C.-L. Ho and Y. Hosotani, Int. J. Mod. Phys. A 7, 5795 (1992); Phys. Rev. Lett. 70, 1360 (1993); D. Wesolowski, Y. Hosotani, and C.-L. Ho, Int. J. Mod. Phys. A 9, 969 (1994).

[9] C.-L. Ho, Mod. Phys. Lett. A 10, 2665 (1995); J. Phys. A 29, L107 (1996).

[10] M.B. Green, J.H. Schwarz, and E. Witten, Superstring Theory, Vol.2 (Cambridge University Press, Cambridge, England, 1987).

[11] A.T. Davies and A.McLachlan, Phys. Lett. 200B , 305 (1988); Nucl. Phys. B 317, 237 (1989); A. Higuchi and L.Parker, Phys. Rev. D 37, 2853 (1988); M. Burgess and D.J. Toms, Phys. Lett. B 234, 97 (1990); C.-L. Ho and K.-W. Ng, Phys. Rev. D 43, R3107 (1991).

[12] J.E. Hetrick and C.-L. Ho, Phys. Rev. D 40, 4085 (1989).

[13] A. McLachlan, Nucl. Phys. 338B , 188 (1990).

[14] K. Shiraishi, Z. Phys. C 35, 37 (1987).

[15] C.-L. Ho and Y. Hosotani, Nucl. Phys. 345B , 445 (1990).

[16] A. McLachlan, Class. Quant. Grav. 7, L103 (1990).

[17] C.-C. Lee and C.-L. Ho, Mod. Phys. Lett. A 8, 1495 (1993).

[18] E. Witten, Nucl. Phys. 258B , 75 (1985).

[19] C. Bernard, Phys. Rev. D 9, 3312 (1974); L. Dolan and R. Jackiw, ibid. 9, 3320 (1974); R.J. Rivers, Path Integral Methods in Quantum Field Theory (Cambridge University Press, Cambridge, 1987); J.I. Kapusta, Finite Temperature Field Theory (Cambridge University Press, Cambridge, 1989).

[20] K. Shiraishi, Prog. Theor. Phys. 77, 1253 (1987).

[21] L. Lewin, Polylogarithms and Associated Functions (North-Holland, New York, 1981). 
[22] B.J. Harrington and A. Yildiz, Phys. Rev. Lett. 33, 324 (1974); T.D. Lee and G.C. Wick, Phys. Rev. D 9, 2291 (1974); T.D. Lee and M. Margulies, ibid. 11, 1591 (1975); J.F. Bolzan and W.F Palmer, ibid. 11, 2932 (1975).

[23] A.D. Linde, Phys. Rev. D 14, 3345 (1976). 


\section{Figure Captions}

Figure 1. Symmetry patterns of the vacuum in an $\mathrm{SU}(2)$ gauge theory on spacetime $R^{1,1} \times$ $T^{2}$ with boundary conditions $\beta_{1}=\beta_{2}=0$ at zero temperature and finite density $\mu$. The phase diagram is plotted as a function of $\mu L_{1}$ and $r=L_{2} / L_{1}$, where $L_{1}$ and $L_{2}$ are the lengths of the torus. Shaded regions represent the symmetric phase, while the unshaded ones represent the broken phase.

Figure 2. Symmetry patterns of the vacuum in an $\mathrm{SU}(2)$ gauge theory on spaectime $R^{1,2} \times$ $S^{1}$ with boundary condition $\beta_{1}=0$ at finite temperature $T$ and density $\mu$. The phase diagram is plotted as a function of $\mu L$ and $T L$, where $L$ is the length of the circle. 